\title{
EVALUACIÓN DE LA EFICACIA DE DOS TERAPIAS ESTROGÉNICAS LOCALES MÁS UN LUBRICANTE VAGINAL, PARA EL CONTROL DE LOS SÍNTOMAS DEL SÍNDROME GENITOURINARIO DE LA MENOPAUSIA
}

Franklin José Espitia De La Hoz' ${ }^{1}$ Hoover Orozco Gallego²

\section{Resumen}

Objetivo: Evaluar la eficacia de dos terapias estrogénicas locales más un lubricante vaginal (K-Y gel), para el control de los síntomas del síndrome genitourinario de la menopausia. Materiales y métodos: Ensayo clínico, aleatorizado, prospectivo y controlado, no enmascarado. Se incluyeron 57 mujeres posmenopáusicas atendidas en la consulta de climaterio, entre julio de 2014 y julio de 2016 . Se asignaron dos grupos aleatorizados: grupo A (30 mujeres recibieron $0.625 \mathrm{mg}$ intravaginales de estrógenos equinos conjugados dos veces a la semana), y grupo B (27 mujeres recibieron $0,5 \mathrm{mg}$ intravaginales de estriol dos veces a la semana) durante seis meses. Ambos grupos recibieron, adicional a la terapia hormonal, 5 gramos intravaginales del lubricante cada 6 horas. A todas las mujeres, para evaluar el estatus hormonal, antes del inicio y a los seis meses, se les determinó el índice de maduración vaginal, y mensualmente se les evaluaba la mejoría de la sintomatología del síndrome genitourinario de la menopausia, además de calcularles el índice de salud vaginal. Resultados: La edad promedio del grupo fue de 58,03 $\pm 4,85$ años. Se observó una significativa disminución en

Palabras clave: Atrofia, Estrógenos, Menopausia, Lubricantes, Vulvovaginitis, Dispareunia

1 Especialista Ginecología y Obstetricia. Universidad Militar Nueva Granada. Máster en Sexología: Educación y asesoramiento sexual. Universidad de Alcalá de Henares. Clínica La Sagrada Familia. Armenia, Quindío, Colombia, Suramérica ${ }^{2}$ Licenciatura en Física y Matemáticas. Universidad Tecnológica De Pereira - UTP. Maestría/Magister en Física. Universidad Tecnológica De Pereira - UTP 
los síntomas en el grupo B, comparado con el grupo A ( $\mathrm{p}<0,001)$, al mes $(p<0,001)$ y a los 6 meses de seguimiento $(p=0,018)$. El tiempo para lograr una mejor puntuación en el índice de salud vaginal, fue significativamente menor en el grupo B. Al final de la investigación, la evaluación de los cambios en el índice de maduración vaginal, mostró un aumento en las células superficiales e intermedias, en el grupo B, superior al grupo A, mientras se reportó mayor disminución en el porcentaje de células parabasales del grupo B. Las mujeres del grupo A mostraron una reducción en la sintomatología, al final del estudio, del $72 \%$ frente al 87\% del grupo B. Conclusiones: El uso de la terapia estrogénica local, en el síndrome genitourinario de la menopausia, a los seis meses de seguimiento, pone de manifiesto una mejoría significativa de los síntomas. El uso de estriol más lubricante, se asoció con mejores resultados y menores efectos adversos, en comparación con los estrógenos equinos conjugados más lubricante. 


\section{EVALUATION OF THE EFFECTIVENESS OF THE COMBINATION OF TWO LOCAL ESTROGENIC THERAPIES WITH A VAGINAL LUBRICANT, FOR THE SYMPTOMS CONTROL OF GENITOURINARY SYNDROME OF MENOPAUSE}

Franklin José Espitia De La Hoz' ${ }^{1}$ Hoover Orozco Gallego²

\section{Abstract}

Objective. To evaluate the efficacy of the combination of two local estrogenic therapies with a vaginal lubricant (K-Y gel), to control the symptoms of the severe atrophic vulvovaginitis. Materials and methods. Prospective, controlled and randomized study. Fifty-seven postmenopausal women treated by the author in the climacteric consultation, between July 2014 and July 2016 were included. Two groups were established, 30 women received twice a week $0.625 \mathrm{mg}$ intravaginal conjugated equine estrogens (group A), and 27 Women received twice a week during six months Estriol at doses of $0.5 \mathrm{mg}$ intravaginal (group B). Both groups received, in addition to the hormonal therapy, 5 grams of intravaginal lubricant every 6 hours. In order to evaluate the hormonal status of all women, before starting and six months later, their vaginal maturity index was determined, simultaneously, the vaginal health index was calculated monthly in each control. Results. The women of group A showed a symptoms reduction, at the end of the study, of $72 \%$ compared to $87 \%$ of group B. None of the women presented complications related to therapy. Conclusions. The use of local estrogen therapy, in severe atrophic vulvovaginitis, is a well-tolerated measure; after six months of monitoring, a significant improvement of the symptoms and the vaginal health index is evidenced. The use of estriol with lubricant was associated with better results and less adverse effects, compared to conjugated equine estrogens and lubricant; however, the rates of sexual satisfaction were similar. 


\section{AVALIAÇÃO DA EFICÁCIA DA COMBINAÇÃO DE DUAS TERÁPIAS COM ESTROGÊNIO LOCAL COM UM LUBRIFICANTE VAGINAL, PARA O CONTROLE DOS SINTOMAS DA SÍNDROME GENITURINÁRIA DA MENOPAUSA}

Franklin José Espitia De La Hoz'1, Hoover Orozco Gallego²

\section{Resumo}

Objetivo. Avaliar a eficácia da combinação de duas terapias com estrogênio local com um lubrificante vaginal (K-Y gel), para o controle dos sintomas da vulvovaginite atrófica grave.

Materiais e métodos. Estudo prospectivo, controlado e randomizado. Foram incluídas 57 mulheres na pós-menopausa que frequentam consulta de climatério do autor, entre julho de 2014 e julho de 2016. Dois grupos foram estabelecidos, 30 mulheres receberam $0,625 \mathrm{mg}$ de estrogénios equinos conjugados, duas vezes por semana por via intravaginal (grupo A), e 27 mulheres receberam estriol em doses de $0,5 \mathrm{mg}$ intravaginalmente duas vezes por semana durante seis meses (grupo B). Ambos os grupos receberam, além da terapia hormonal, 5 gramas de lubrificante intravaginal a cada 6 horas. A fim de avaliar o estado hormonal, antes do início e aos seis meses, determinou-se o índice de maturidade vaginal, para todas as mulheres, calculando-se simultaneamente o índice de saúde vaginal, que foi continuado mensalmente em cada controle.

Resultados. As mulheres do grupo A apresentaram redução da sintomatologia, ao final do estudo, de $72 \%$ contra $87 \%$ do grupo B. Nenhuma das mulheres apresentou complicações relacionadas à terapia.

Conclusões. O uso de terapia de estrogênio local, na vulvovaginite atrófica grave, é uma medida bem tolerada; aos seis meses de acompanhamento, é evidente uma melhora significativa dos sintomas e do índice de saúde vaginal. O uso de estriol com lubrificante, foi associado com melhores resultados e menos efeitos adversos, comparado aos estrogênios e lubrificantes equinos conjugados; no entanto, as taxas de satisfação sexual foram semelhantes. 


\section{Introducción}

Las manifestaciones de los trastornos tisulares del aparato urogenital y del piso pélvico, se hacen más evidentes después de la llegada de la menopausia, tornándose más severos con el progresivo envejecimiento $(1,2)$.

La atrofia vulvovaginal es a menudo descrita como el "síntoma silencioso" de la menopausia, y hoy ha sido reemplazada por una nueva terminología: síndrome genitourinario de la menopausia (SGUM) (2). Se estima que entre el 10$40 \%$ de las mujeres posmenopáusicas se ven afectadas, sin embargo, sólo alrededor del 25\% de las sintomáticas buscan atención médica, siendo pocas las que reciben terapia hormonal $(2,3)$. Esta condición puede tener un gran impacto en la calidad de vida y en la función sexual de estas mujeres, pero a menudo es infradiagnosticada $y$, por ende, tratada con deficiencia. A diferencia de los sín- tomas vasomotores, que normalmente disminuirán con el tiempo, los síntomas vulvovaginales del síndrome genitourinario de la menopausia son progresivos y menos propensos a resolver sin tratamiento eficaz $(2,4,5)$.

La sintomatología relacionada con el envejecimiento urogenital se podría dividir en dos grupos: los relacionados en el tracto urinario inferior (uretra y vejiga) y los que involucran directamente a la vagina y a la vulva (tabla 1). En el primer grupo se destacan: disuria, frecuencia y urgencia urinaria, incontinencia urinaria, infecciones recurrentes y nicturia; en el segundo grupo sobresalen: ardor, canal vaginal estrecho y poco distensible, disminución de la lubricación, disminución del tamaño del clítoris, dispareunia, escozor y prurito vulvar, fusión de los labios menores y sequedad vaginal (tabla 2); los que son causados por la pérdida de la producción de estrógenos $(2,3,6,7)$.

Tabla No. 1. Sintomatología del síndrome genitourinario de la menopausia

\begin{tabular}{ll}
\hline \multicolumn{1}{c}{ Síntomas vulvovaginales } & \multicolumn{1}{c}{ Síntomas del tracto urinario inferior } \\
\hline Ardor & Disuria \\
Dispareunia & Nicturia \\
Dolor & Polaquiuria \\
Prurito & Urgencia urinaria \\
Disminución de la lubricación vaginal & Tenesmo vesical \\
Molestias coitales & Infecciones urinarias recidivantes \\
Quemazón & Incontinencia urinaria \\
Sangrado postcoital & Infección postcoital \\
Sequedad vaginal & Disminución del flujo de orina uretral \\
\hline
\end{tabular}


Los hallazgos vulvovaginales manifiestos con la atrofia urogenital, en las mujeres posmenopáusicas, se valoran con el índice vaginal de salud [en inglés: $V a-$ ginal Health Index Score (VHIS)]. Cada parámetro se puntúa del 1 al 5, considerándose atrofia vaginal severa valores por debajo del 15 , por lo tanto, a menor puntuación, mayor grado de atrofia. Este índice emplea datos objetivos, estimables durante la evaluación ginecológica; siendo un parámetro útil para evaluar la intensidad de los síntomas, gravedad de la atrofia y la respuesta al tratamiento (8), datos utilizados en esta investigación para el seguimiento mensual de la eficacia de la terapia.

Tabla No. 2. Signos clínicos del síndrome genitourinario de la menopausia

\begin{tabular}{|c|c|c|}
\hline Vulvares & Vaginales & Urinarios \\
\hline $\begin{array}{l}\text { Pérdida del vello púbico o } \\
\text { escaso y fino. }\end{array}$ & $\begin{array}{l}\text { Paredes vaginales pálidas, } \\
\text { secas y acartonadas. }\end{array}$ & $\begin{array}{l}\text { Aumento del residuo post- } \\
\text { miccional. }\end{array}$ \\
\hline $\begin{array}{l}\text { Pérdida de la almohadilla } \\
\text { adiposa labial. }\end{array}$ & $\begin{array}{l}\text { Alisamiento de los fondos de } \\
\text { saco vaginales. }\end{array}$ & $\begin{array}{l}\text { Disminución de la capacidad } \\
\text { vesical. }\end{array}$ \\
\hline $\begin{array}{l}\text { Retracción y pérdida de } \\
\text { la definición de los labios } \\
\text { mayores y menores. }\end{array}$ & $\begin{array}{l}\text { Adelgazamiento de la } \\
\text { superficie vaginal. }\end{array}$ & $\begin{array}{l}\text { Disminución del umbral de } \\
\text { sensibilidad a la distensión de } \\
\text { la vejiga. }\end{array}$ \\
\hline $\begin{array}{l}\text { Acortamiento del prepucio } \\
\text { y exposición excesiva del } \\
\text { clítoris. }\end{array}$ & $\begin{array}{l}\text { Petequias, fisuras, ulceración e } \\
\text { inflamación. }\end{array}$ & $\begin{array}{l}\text { Disminución de la presión de } \\
\text { cierre uretral. }\end{array}$ \\
\hline $\begin{array}{l}\text { Disminución de la sensibilidad } \\
\text { vestibular. }\end{array}$ & $\begin{array}{l}\text { Leucorrea y/o secreción } \\
\text { anormal. }\end{array}$ & Meato uretral prominente. \\
\hline $\begin{array}{l}\text { Estrechamiento del introito } \\
\text { vaginal. }\end{array}$ & Pérdida de la elasticidad. & $\begin{array}{l}\text { Índice de maduración uretral } \\
\text { anormal. }\end{array}$ \\
\hline $\begin{array}{l}\text { Fusión de los labios y/o } \\
\text { pérdida de la integridad. }\end{array}$ & $\begin{array}{l}\text { Pérdida de los pliegues y } \\
\text { rugosidades vaginales. }\end{array}$ & $\begin{array}{l}\text { Disminución de la presión } \\
\text { máxima de contracción } \\
\text { miccional del músculo } \\
\text { detrusor. }\end{array}$ \\
\hline Flujo sanguíneo disminuido. & Disminución de la humedad. & Prolapso uretral, \\
\hline $\begin{array}{l}\text { Susceptibilidad a traumas } \\
\text { mecánicos. }\end{array}$ & $\begin{array}{l}\text { Adelgazamiento del epitelio } \\
\text { vaginal. }\end{array}$ & \\
\hline Retracción del clítoris. & $\begin{array}{l}\text { Aumento sobre } 4.6 \text { del pH } \\
\text { vaginal. }\end{array}$ & \\
\hline \multirow[t]{3}{*}{$\begin{array}{l}\text { Piel hipopigmentada, lisa, no } \\
\text { elástica y/o brillante. }\end{array}$} & $\begin{array}{l}\text { Cambios en la flora facultativa } \\
\text { de la vagina. }\end{array}$ & \\
\hline & $\begin{array}{l}\text { Acortamiento, fibrosis, } \\
\text { obliteración de la cúpula } \\
\text { vaginal. }\end{array}$ & \\
\hline & $\begin{array}{l}\text { Cambios en el índice de } \\
\text { maduración vaginal (IMV). }\end{array}$ & \\
\hline
\end{tabular}

Fuente: Elaboración propia. 
Las opciones terapéuticas para el tratamiento del SGUM son diversas. Las alternativas farmacológicas se suelen dividir en dos vertientes: no hormonales u hormonales. Entre los no hormonales los hidratantes y los lubricantes vaginales constituyen la primera línea terapéutica para aliviar los síntomas [Nivel de evidencia A] $(2,5,9)$. El objetivo de la terapia se basa en la reducción de los síntomas y la restauración de los tejidos afectados; resultando la terapia local con estrógenos, el tratamiento de elección en aquellas mujeres con atrofia vulvovaginal que carecen de otros síntomas climatéricos (2,9-11).

La terapia local con estrógenos promueve la revascularización y engrosamiento del epitelio vaginal y del tracto urinario inferior, resultando en una mayor lubricación, mejora de la elasticidad y disminución del $\mathrm{pH}$ vaginal, con efectos positivos sobre el índice de maduración vaginal $(2,7,8,12)$.

Son varias las preparaciones de estrógenos locales que han demostrado disminuir los síntomas de la atrofia, incluyendo la sequedad vaginal, irritación, prurito, dispareunia y urgencia urinaria $(2,3,13,14)$. Estos tratamientos también aumentan el flujo sanguíneo y la lubricación, lo que puede influir positivamente en la función sexual $(2,15)$.

Los lubricantes se suelen utilizar para aumentar el placer durante la relación sexual, además de disminuir la fricción y la dispareunia; sin embargo, esta no es la única indicación que tienen, ya que la aplicación regular de hidratantes y lubricantes, son una opción terapéutica en mujeres con SGUM que no desean utilizar tratamientos hormonales $(2,8)$, pudiendo utilizarse de forma rutinaria e indefinida (2).

En la actualidad el uso de los estrógenos vaginales, estradiol y estrógenos conjugados, han sido aprobados para el tratamiento de la atrofia vulvovaginal, sequedad vaginal y dispareunia $(2,5,7)$. El estriol ha mostrado cambios positivos en el índice de maduración vaginal, el $\mathrm{pH}$ vaginal, sequedad vaginal y la puntuación global de los síntomas $(2,15,16)$. Los lubricantes a base de agua proporcionan lubricación para mejorar la sequedad vaginal, concediendo mayor comodidad durante la actividad sexual, aunque no suministran humedad a largo plazo (17), además de no revertir la atrofia vulvovaginal, pero pueden aportan un confort adicional a corto plazo, durante y después de la actividad sexual (2).

Los lubricantes a base de agua tienen propiedades adicionales, como que no erosionan los preservativos al no dañar el látex (18-20); siendo recomendados como la primera línea terapéutica para el tratamiento de los síntomas leves o moderados de la vulvovaginitis atrófica (21).

Luego de una exhaustiva revisión de las diferentes investigaciones y publicaciones realizadas con relación al tratamiento del SGUM, nuestro equipo de trabajo se planteó la necesidad de estudiar la eficacia de la combinación de dos terapias estrogénicas locales con un lubricante vaginal en el tratamiento de los 
publicaciones al respecto. El objetivo de este estudio es evaluar la eficacia de la combinación de dos terapias estrogénicas locales con un lubricante vaginal (K-Y gel), para el control de los síntomas del SGUM, así como determinar el índice de maduración vaginal y el índice de salud vaginal, así como conocer los posibles efectos adversos en mujeres atendidas en la Clínica La Sagrada Familia, en la ciudad de Armenia.

\section{Material y métodos}

Se realizó un estudio unicéntrico, prospectivo, aleatorizado y controlado, no enmascarado. Se eligieron mujeres con diagnóstico clínico de SGUM, que asistieron a la consulta del autor, en el periodo del 1 de julio de 2014 y el 31 de julio de 2016, en la Clínica La Sagrada Familia, una institución privada, de alta complejidad, en la ciudad de Armenia, que atiende población perteneciente al régimen contributivo y subsidiado. El diagnóstico se hizo mediante la exploración clínica de los síntomas y signos de la menopausia asociados con cambios físicos de la vulva, la vagina y el tracto urinario inferior de acuerdo a las tablas 1 y 2.

Entre los criterios de inclusión se tuvieron en cuenta la edad mayor de 51 años, no menos de cinco años de la fecha de la última menstruación (FUR), no haber recibido tratamiento hormonal seis meses antes, no tener contraindicación para la terapia hormonal y estar histerectomizadas. Entre los criterios de exclusión se consideraron infección vaginal activa, citología cervical alterada, antecedente de cáncer o estar en tratamiento de radioterapia o quimioterapia.
Las mujeres candidatas a ingresar al estudio fueron identificadas por el autor en la consulta externa. Luego de la valoración y de la verificación de los criterios de inclusión y exclusión, se invitaron a participar. Se les explicó el objetivo de la investigación y se solicitó su autorización para ser incluidas en el estudio mediante la firma del consentimiento informado.

Las mujeres fueron asignadas de forma aleatoria a uno de dos grupos, utilizando una tabla de números aleatorios construida mediante un código de asignación generado por un algoritmo computacional, preparados por un observador ajeno al estudio. Se asignaron dos grupos de tratamiento: grupo (A) las que recibieron $0.625 \mathrm{mg}$ intravaginales de estrógenos equinos conjugados dos veces a la semana y el otro grupo (B) que recibieron estriol a dosis de $0,5 \mathrm{mg}$ intravaginales dos veces a la semana. Ambos grupos recibieron la terapia durante seis meses, y adicional a la terapia hormonal, se les suministró 5 gramos intravaginales del lubricante (K-Y gel) cada 6 horas.

No se realizó un cálculo del tamaño de muestra, sino que se hizo un muestreo por conveniencia a partir del universo de mujeres atendidas en la institución participante durante el periodo del estudio.

La maniobra de intervención consistió en comparar la eficacia de la combinación de dos terapias estrogénicas locales: estrógenos equinos conjugados y estriol con un lubricante vaginal (K-Y gel), en el control de los síntomas del SGUM. Así como evaluar, el índice de madura- 
ción vaginal y el índice de salud vaginal, así como la presencia de eventuales efectos adversos.

A todas las mujeres se les determinó el índice de maduración vaginal, para evaluar el estatus hormonal, al inicio de la terapia y a los seis meses (luego de finalizado el estudio); se les tomó la muestra con un rascado suave a lo largo de la pared lateral de la vagina superior, además se les calculaba mensualmente el índice de salud vaginal (en cada control) y se les interrogaba la presencia de efectos adversos.

A todas las mujeres se les hizo una valoración inicial que incluía la evaluación de los síntomas somáticos, antecedentes médicos y quirúrgicos, un examen físico y ginecológico completo; se les evaluaba el $\mathrm{pH}$ vaginal, se les realizaba una colposcopia, frotis vaginal y se les calculaba el índice de salud vaginal mensualmente en cada control, además de calcularles el índice de maduración vaginal al ingreso $\mathrm{y}$ a los seis meses, una vez finalizado el estudio. Todas las mujeres presentaban síntomas y signos relacionados con el SGUM de acuerdo a las tablas 1 y 2 .

Se realizó una tabla de Excel donde se recogían los datos, incluyendo la información clínica y quirúrgica más relevante de las mujeres del estudio, así como los efectos secundarios asociados a la terapia. Junto a ello se objetivaron los cambios en el índice de maduración y del índice de salud vaginal.

\section{Análisis estadístico}

El estudio estadístico se realizó con el programa estadístico SPSS versión 15.0
(SPSS, Chicago, Illinois. EEUU). Se utilizó estadística descriptiva para resumir la información de las variables continuas mediante medida de tendencia central y dispersión según normalidad y proporciones para variables categóricas. Los dos grupos de intervención fueron comparados por medio del estadístico $\chi 2$ de Pearson en variables categóricas y la prueba t de Student para variables continuas con un nivel de significancia del 0.05 .

Aspectos éticos. Esta investigación contó con la aprobación del comité de ética de la Clínica La Sagrada Familia, y el consentimiento informado de las mujeres participantes; se garantizó la confidencialidad de las pacientes.

\section{Resultados}

En el momento del cierre del periodo de reclutamiento de las mujeres del estudio, se había incluido a un total de 66 mujeres, que fueron aleatorizadas entre enero de 2009 a diciembre de 2015. En el grupo $A(n=34)$ tres pacientes y en el grupo $\mathrm{B}(\mathrm{n}=32)$ dos pacientes, fueron excluidas antes del inicio de la terapia porque se negaron a firmar el consentimiento informado. En tres pacientes hubo la necesidad de realizar tratamiento quirúrgico para prolapso genital (una del grupo A y dos del grupo B). Una paciente del grupo $\mathrm{B}$ decidió únicamente utilizar lubricante. Estas 9 pacientes no se incluyeron en el análisis. Al final se analizaron 57 mujeres diagnosticadas con SGUM, distribuidos en dos grupos A ( $\mathrm{n}=30$ mujeres) y B ( $n=27$ mujeres). No se produjo ninguna pérdida en el seguimiento de 
de las 57 pacientes fueron similares a las del resto de las mujeres del estudio.

Las características sociodemográficas de ambos grupos se muestran en la tabla 3. La edad promedio de las pa- cientes fue de 58,03 $\pm 4,85$ años (rango entre 51 y 87 años); siendo el grupo de más frecuencia el de 60 a 70 años. No se documentaron diferencias en las medidas antropométricas y características sociodemográficas.

Tabla 3. Características sociodemográficas en mujeres con síndrome genitourinario de la menopausia

\begin{tabular}{|c|c|c|c|}
\hline & Grupo A $(n=30)$ & Grupo B $(n=27)$ & Valor $p$ \\
\hline Edad, $X \pm D E$ & $53 \pm 6.4$ & $54 \pm 7.8$ & 0.87 \\
\hline Peso, $X \pm D E$ & $64.5 \pm 7.5$ & $65.7 \pm 8.1$ & 0.75 \\
\hline Talla, X $\pm D E$ & $1.59 \pm 0.27$ & $1.62 \pm 0.3$ & 0.66 \\
\hline$I M C, X \pm D E$ & $27.9 \pm 8.7$ & $29.1 \pm 4.5$ & 0.78 \\
\hline \multicolumn{4}{|l|}{ Raza } \\
\hline Blanca & 21 & 18 & 0.28 \\
\hline Afrocolombiana & 3 & 4 & 0.81 \\
\hline Hispánica & 6 & 5 & 0.33 \\
\hline Edad promedio de menopausia (años) & $47.1 \pm 4.5$ & $43.5 \pm 3.9$ & 0.36 \\
\hline Años en posmenopausia & $5.9 \pm 7.3$ & $6.3 \pm 8.7$ & 0.81 \\
\hline Paridad $X \pm D E$ & $3.5 \pm 2.8$ & $2.7 \pm 2.4$ & 0.36 \\
\hline Unión libre, n (\%) & 40.5 & 32.7 & 0.11 \\
\hline Casadas, n (\%) & 38.7 & 32.1 & 0.15 \\
\hline Fumadoras, n (\%) & 10.5 & 7.2 & 0.24 \\
\hline Ingesta alcohol & 31.5 & 36.6 & 0.27 \\
\hline 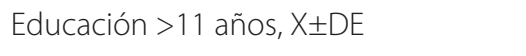 & $11.7 \pm 5.7$ & $12.9 \pm 5.1$ & 0.96 \\
\hline Amas de casa, n (\%) & 43.8 & 37.8 & 0.25 \\
\hline Profesionales, n (\%) & 29.7 & 23.7 & 0.13 \\
\hline Urbana, n (\%) & 78.9 & 80.7 & 0.75 \\
\hline Cirugía ginecológica / urológica, n (\%) & 63.9 & 59.4 & 0.36 \\
\hline Heterosexual, n (\%) & 82.5 & 80.4 & 0.72 \\
\hline
\end{tabular}

En el grupo A, 13 pacientes $(43,3 \%)$ y en el grupo B, 14 pacientes $(51,8 \%)$ tenían síntomas de sangrado postcoital e infecciones urinarias recidivantes. En el grupo A, 3 pacientes $(13,3 \%)$ y en el grupo B, 5 pacientes $(18,5 \%)$ tenían ardor y nicturia. Las infecciones urinarias fueron más frecuentes en el grupo A, sin diferencia estadísticamente significativa ( $90 \%$ vs $88 \%$; $\mathrm{p}=0,07$ ); mientras que, en 
la población global, del total de las 57 mujeres, el 56,1\% $(n=32)$ experimentaron afectación de su función sexual.

Antes del inicio de la terapia, 11 (36,6\%) eran sexualmente activas en el grupo A e informaron dispareunia el 81,8 (9); en el grupo B, $12(44,4 \%)$ eran sexualmente activas, con 91,6 (10) que informaron dispareunia.
La Tabla 4 resume los síntomas y signos que se encontraron en las pacientes, en cada grupo. La sequedad vaginal fue más frecuente en ambos grupos: 92\% y $96 \%$, respectivamente, así como la dispareunia ( $96 \%$ y $82,6 \%$, respectivamente). Entre los síntomas urinarios la disuria y la urgencia urinaria fueron las predominantes.

Tabla 4. Síntomas y signos en mujeres con síndrome genitourinario de la menopausia, en la Clínica La Sagrada Familia

\begin{tabular}{lccc}
\hline & $\begin{array}{c}\text { Estrógenos } \\
\text { equinos } \\
\text { conjugados más } \\
\text { lubricante } \\
\text { (Grupo A) }\end{array}$ & $\begin{array}{c}\text { Estriol más } \\
\text { lubricante } \\
\text { (Grupo B) }\end{array}$ & p \\
\hline Muestra & $\mathrm{n}=30$ & $\mathrm{n}=27$ & \\
Sequedad vaginal & $93,3 \%(28)$ & $85,1 \%(23)$ & 0,04 \\
Dispareunia & $96,6 \%(29)$ & $81,4 \%(22)$ & 0,03 \\
Prurito & $83,3 \%(25)$ & $77,7 \%(21)$ & 0,05 \\
Urgencia urinaria & $76,6 \%(23)$ & $74,1 \%(20)$ & $\mathrm{NS}$ \\
Infección postcoital & $46,6 \%(14)$ & $40,7 \%(11)$ & 0,005 \\
Disuria & $63,3 \%(19)$ & $62,9 \%(17)$ & \\
Prolapso uretral & $36,6 \%(11)$ & $37,4 \%(10)$ & \\
Meato uretral prominente & $26,6 \%(8)$ & $29,6 \%(8)$ & \\
Adelgazamiento de la superficie & $86,6 \%(26)$ & $88,8 \%(24)$ & \\
vaginal & $23,3 \%(7)$ & $29,6 \%(6)$ & \\
Fimosis del prepucio del clítoris & & & \\
\hline
\end{tabular}

La puntuación del índice de maduración vaginal, en la población global, del total de las 57 mujeres fue de $21 \pm 6$ puntos; siendo el puntaje mayor de 34 y el mínimo de 13, con una desviación estándar de \pm 5 .

Al comparar el índice de maduración vaginal, se comprobó que, entre los dos grupos, hubo una diferencia estadística- mente significativa a los seis meses de la terapia, $54 \pm 3$ en el grupo A y $81 \pm 9$ en el grupo $B(p=0,04)$; se pudo comprobar que, en el grupo de la terapia con estrógenos equinos conjugados más lubricante, los puntajes eran inferiores respecto al grupo al que se les administró estriol más lubricante. 
La puntuación del índice de salud vaginal, en la población global, del total de las 57 mujeres fue de 9,5 $\pm 3,4$; siendo el puntaje mayor de 14 y el mínimo de 7 , con una desviación estándar de \pm 4 . El tiempo para lograr una mejor puntuación en el índice de salud vaginal, fue significativamente mayor, en el grupo A, con una puntuación media a los seis meses de $11,4 \pm 0,42$ frente al 15,9 $\pm 0,72$ del grupo $B(p=0,04)$.

\begin{abstract}
Al final de la investigación, la evaluación de los cambios en el índice de maduración vaginal, mostró un aumento en las células superficiales e intermedias de 8,4 a 57,9\% en el grupo A y de 9,51 a 76,3\% en el grupo $B(p=0,013$. El porcentaje de células parabasales disminuyó de 71,4 a $7,1 \%$ en el grupo A y de 77,1 a $4,35 \%$ en el grupo $B(p=0,002)$.
\end{abstract}

La mejoría de la sequedad vaginal, fue manifiesta en ambos grupos después de las 9 semanas, pero mucho mejor luego de las 15 semanas, y significativamente superior en el grupo del estriol con lubricante (pasó de $57,5 \%$ a $77,5 \%$; $\mathrm{p}=0,004$ ); evidenciándose, a corto plazo, la adecuada recuperación de las características fisiológicas de la mucosa vaginal con ambas terapias $(\mathrm{p}=0,384)$.

La mejoría de la dispareunia en las primeras 9 semanas, fue significativamente mayor en el grupo B ( $86,9 \%$ frente a $62,5 \%, p=0,0035)$, diferencia que se mantuvo en el tiempo hasta el final del estudio, y la mejoría en la lubricación vaginal fue significativamente mayor en el grupo B $(82,7 \%$ frente a $72,4 \%$, $\mathrm{p}=0,031$ ); lo cual fue relevante a la hora de presentar menor dispareunia a la pe- netración. Las mujeres que refirieron estrechamiento del introito vaginal antes del inicio de la terapia, ninguna la manifestó al final del estudio, en ambos grupos.

La mejoría y la presencia de cambios macroscópicos positivos en la vagina, se observó en el 55,5\% de las mujeres del grupo B y en el $40 \%$ del grupo A, a las 4 semanas $(p<0,001)$. Se presentó una significativa disminución en los síntomas urinarios en el grupo $\mathrm{B}$, comparado con el grupo $A(p<0,001)$, al mes $(p<0,001)$ y a los 6 meses de seguimiento $(p=0,018)$.

La tasa de satisfacción sexual inmediata al uso de la terapia (primeras dos semanas), relacionadas con ambas terapias fue similar (70\% del grupo A frente al $77,7 \%$ del grupo $\mathrm{B}, \mathrm{p}=0,1)$, manteniéndose en el tiempo hasta el final del estudio.

Tras una media de seguimiento de tres meses, el índice de salud vaginal mostró una mejoría del 43,3\% en el grupo de estrógenos equinos conjugados más lubricante frente al 59,2\% en el grupo de estriol más lubricante $(\mathrm{p}=0,041)$. Sobre la estrechez vaginal no existió efecto alguno con ambas terapias $(\mathrm{p}=0,24)$, pero si sobre el adelgazamiento de la superficie vaginal. Por tanto, el beneficio se centra en la sintomatología. Sin embargo, no hubo diferencias en la retracción del clítoris, en ambos grupos (36,6 vs. 40,7\%) ni tampoco en la incontinencia urinaria (24 vs. $26,6 \%),(p=0,205)$.

En relación con los efectos adversos, 6 mujeres experimentaron dolor mamario en el grupo de estrógenos equinos conjugados frente a 2 mujeres en el 
grupo de estriol $(p<0,01)$, 9 mujeres experimentaron aumento de la secreción vaginal frente a $5(\mathrm{p}<0,01)$ y 3 mujeres experimentaron irritación vaginal frente a $1(\mathrm{p}<0,01)$. Ninguna de esta sintomatología condujo a la suspensión de la terapia por parte de las pacientes de ambos grupos. Es necesario tener presente que las mujeres de este estudio, estaban en seguimiento continuo, -se les hacía controles periódicos (meses 1 , $2,3,4,5$ y 6$)$-.

Para la mayoría de las mujeres en nuestro estudio, esta fue su primera terapia hormonal (73,3\% grupo A y 74,1\% grupo B). El incremento de la actividad coital fue de 1 a 3 encuentros por mes, con una mediana de 2 coitos por mes, para el grupo A; y de 1 a 4 encuentros por mes para el grupo B, con una mediana de 3 coitos por mes. Estas variables no muestran diferencias estadísticamente significativas $(\mathrm{p}=0,889$ y $\mathrm{p}=0,894$, respectivamente).

La combinación estriol más lubricante, mostró una ventaja clínica estadísticamente significativa sobre la combinación estrógenos equinos conjugados más lubricante $(\mathrm{p}=0,002)$, puesto que hubo más reducción de los síntomas irritativos del tracto urinario inferior característicos del SGUM en menor tiempo.

El estudio concluye que la eficacia de la combinación de estrógenos conjugados más lubricante, para el control de los síntomas del síndrome genitourinario de la menopausia, fue inferior a la de estriol más lubricante ( $72 \%$ vs $87 \%, \mathrm{p}=$ $0,023)$.

\section{Discusión}

El síndrome genitourinario de la menopausia es una afección vulvovaginal crónica y progresiva con afectación del tracto urinario inferior, poniendo en riesgo la calidad de vida y la actividad sexual. Se caracteriza por signos y síntomas que son secundarios a un estado clínico hipoestrogénico, propio de la menopausia; los cuales, a diferencia de otros síntomas de la menopausia, empeoren con el tiempo, siendo poco probable que se resuelvan sin tratamiento $(2,22,23)$.

En esta investigación se encontró que la terapia estrogénica local vaginal y los lubricantes son una adecuada elección en el tratamiento del SGUM, lo cual es concordante con lo publicado por otros autores $(22,24)$; y al tener escasos efectos adversos, debería seguir siendo la terapia de elección, tal como lo han informado otros autores (25-28); ya que adicionalmente, son muchas las mujeres las que no descontinúan o no abandonan el tratamiento, tanto por la significativa mejoría como a los escasos efectos secundarios, tal como se pudo demostrar.

En este estudio se ha demostrado que los estrógenos conjugados de origen equino y el estriol usados de forma local con un lubricante, son efectivos para controlar los síntomas del síndrome genitourinario de la menopausia. Sin embargo, el resultado es más favorable con el estriol ( $87 \%$ vs $72 \%, p=0,02)$, ya que tanto el índice de maduración vaginal como el índice de salud vaginal, arrojaron mayores puntajes; efectividad que ha sido des- 
La mejoría de la sequedad vaginal, fue manifiesta en ambos grupos después de las 9 semanas, pero mucho mejor luego de las 15 semanas, y significativamente superior en el grupo del estriol con lubricante $(\mathrm{p}=0,004)$; evidenciándose, a corto plazo, la adecuada recuperación de las características fisiológicas de la mucosa vaginal con ambas terapias ( $\mathrm{p}=0,384)$, lo que coincide con otros estudios $(4,23,31,32)$. Además del positivo efecto del lubricante, los cuales han revelado mayor eficacia que el placebo, pero menos que las terapias estrogénicas (33), no obstante, cuando se utilizan regularmente como en esta investigación, ofrecen una respuesta satisfactoria (32).

Respecto a los efectos adversos, las publicaciones recomiendan que el estriol es un tratamiento conveniente, seguro y eficaz para la atrofia vaginal posmenopáusica $(23,31,32)$, tal como lo han reportado los presentes resultados.

Los autores no encontraron ensayos clínicos que comparen la eficacia de la terapia estrogénica local con un lubricante (a las dosis empleadas en esta investigación); sin embargo, es evidente e incuestionable, que ambas terapias resultaron eficaces y seguras, con restauración de la fisiología vaginal, tal como ha sido descrito en otras publicaciones (34-37), reconociendo la superioridad de la combinación estriol más lubricante en la presente investigación.

La elevada prevalencia de la sintomatología del SGUM determinada en este estudio, así como los bajos índices de tratamiento, sugieren que las mujeres de esta zona se encuentran en desam- paro de un adecuado seguimiento de su condición postmenopáusica hipoestrógenica. Los resultados son consistentes con los reportados en otros lugares, en los cuales los porcentajes oscilan entre el $63 \%$ y el $75 \%(38,39)$.

Entendemos que los resultados aquí publicados resultan alentadores, por lo tanto, invitamos a realizar la experiencia en un número más significado de mujeres, cuya efectividad sea evaluada con el índice de maduración vaginal o del índice de salud vaginal.

El presente estudio tiene como fortalezas el tipo de combinación de las terapias y los índices utilizados para el seguimiento. Como limitaciones, lo pequeño de la muestra, el hecho de que se realizó en una sola institución; y que además no se evaluaron los niveles séricos de estradiol.

\section{Conclusiones}

Este estudio muestra que el tratamiento con estriol combinado con un lubricante, tiene mayor eficacia que la combinación de estrógenos equinos conjugados más lubricante, para el control de los síntomas del SGUM ( $87 \%$ vs $72 \%$, respectivamente), además de presentar menos efectos adversos. Por lo tanto, su incorporación en la práctica habitual exige que se haga la apropiada individualización de la terapia, por parte del clínico, lo que supone un beneficio excepcional en las pacientes adecuadas.

\section{Conflicto de intereses}

Los autores declaran no tener ningún conflicto de intereses. 


\section{Agradecimientos}

A todas las mujeres que aceptaron participar en esta investigación, ya que gracias a su apoyo fue posible la rea- lización de este estudio, a la doctora Adriana Patricia Camero Lascano por sus oportunas sugerencias en la elaboración del análisis definitivo de los resultados.

\section{Referencias}

1 Lovatsis D, Drutz HP. The role of estrogen in female urinary incontinence and urogenital aging: a review. Ostomy Wound Manage. 1998;44(6):48-53

2 Espitia, De La Hoz, FJ, Orozco, Gallego, $\mathrm{H}$. Abordaje diagnóstico y terapéutico del síndrome genitourinario en la menopausia; actualización. 2017;11(2):67-84.

3 North American Menopause Society. The role of local vaginal estrogen for treatment of vaginal atrophy in postmenopausal women: 2007 position statement of The North American Menopause Society. Menopause. 2007;14(3 Pt 1):355-69; quiz 370-1. doi: 10.1097/gme.0b013e31805170eb

4 Cardozo L, Bachmann G, McClish D, Fonda D, Birgerson L. Meta-analysis of estrogen therapy in the management of urogenital atrophy in postmenopausal women: second report of the Hormones and Urogenital Therapy Committee. Obstet Gynecol. 1998;92(4 Pt 2):722-7.

5 Calleja-Agius J, Brincat MP. Urogenital atrophy. Climacteric. 2009;12(4):279-85. doi: 10.1080/13697130902814751.

6 Versi E, Cardozo L, Brincat M, Cooper D, Montgomery J, Studd J. Correlation of urethral physiology and skin collagen in postmenopausal women. $\mathrm{Br} \mathrm{J}$ Obstet Gynaecol. 1988;95(2):147-52. doi: 10.1111/ j.1471-0528.1988.tb06844.x

7 Nachtigall L, Nachtigall M, Goren J, Loewenstein J. Update on vaginal atrophy. Menopause Manag 2005;17-19.
8 Bachmann GA, Nevadunsky NS. Diagnosis and treatment of atrophic vaginitis. Am Fam Physician. 2000;15;61(10):3090-6.

9 Castelo-Branco C, Cancelo MJ, Villero J, Nohales F, Juliá MD. Management of post-menopausal vaginal atrophy and atrophic vaginitis. Maturitas. 2005;15;52 Suppl 1:S46-52. Epub 2005 Sep 1. doi: 10.1016/j.maturitas.2005.06.014

10 Krychman ML. Vaginal estrogens for the treatment of dyspareunia. J Sex Med 2011;8(3):666-674. doi:10.1111/j.17436109.2010.02114.x

11 North American Menopause Society. Estrogen and progestogen use in postmenopausal women: 2010 position statement of The North American Menopause Society. Menopause. 2010;17(2):242-55. doi: 10.1097/gme.0b013e3181d0f6b9

12 Long CY, Liu CM, Hsu SC, Wu $\mathrm{CH}$, Wang $\mathrm{CL}$, Tsai EM. A randomized comparative study of the effects of oral and topical estrogen therapy on the vaginal vascularization and sexual function in hysterectomized postmenopausal women. Menopause. 2006;13(5):737-43. doi: 10.1097/01. gme.0000227401.98933.0b

13 Simon J, Nachtigall L, Gut R, Lang E, Archer DF, Utian W. Effective treatment of vaginal atrophy with an ultra-low-dose estradiol vaginal tablet. Obstet Gynecol. 2008;112(5):1053-60. doi: 10.1097/AOG. 0b013e31818aa7c3 
14 Nappi RE, Davis SR. The use of hormone therapy for the maintenance of urogynecological and sexual health post WHI. Climacteric. 2012;15(3):267-74. doi: 10.3109/13697137.2012.657589

15 Lee A, Kim TH, Lee HH, Kim YS, Enkhbold T, Lee B, Park YJ, Song K. Therapeutic Approaches to Atrophic Vaginitis in Postmenopausal Women: A Systematic Review with a Network Meta-analysis of Randomized Controlled Trials. Menopausal Med. 2018;24(1):1-10. doi: 10.6118/ jmm.2018.24.1.1

16 Cano A, Estévez J, Usandizaga R, Gallo JL, Guinot M, Delgado JL, et al. The therapeutic effect of a new ultra low concentration estriol gel formulation $(0.005 \%$ estriol vaginal gel) on symptoms and signs of postmenopausal vaginal atrophy: results from a pivotal phase III study. Menopause. 2012;19(10):1130-9. doi: 10.1097/ gme.0b013e3182518e9a

17 Hickey M, Saunders C, Partridge A, Santoro N, Joffe H, Stearns V. Practical clinical guidelines for assessing and managing menopausal symptoms after breast cancer. Ann Oncol. 2008;19(10):1669-80. doi: 10.1093/annonc/mdn353

18 Espitia, De La Hoz, FJ. Mi primer año en La Crónica. Bogotá. Editorial Bolívar. Primera edición. 2016; 5(3):71-72.

19 Espitia, De La Hoz, FJ. Sexo llimitado / El vuelo hacia una sexualidad más placentera. Bogotá. Editorial Bolívar. Primera edición. 2015;2(15):181-185.

20 Palacios S, Cancelo M, Castelo-Branco C, González S, et al. Recomendaciones de la Sociedad Española de Ginecología y Obstetricia sobre la prevención y el tratamiento de la atrofia vaginal. Prog Obstet Ginecol 2012;55(8):408-415

21 Rees M, Pérez-López FR, Ceasu I, 182 Depypere H, Erel T, Lambrinoudaki I, et al. EMAS clinical guide: low-dose vaginal estrogens for postmenopausal vaginal atrophy. Maturitas. 2012;73(2):171-4. doi: 10.1016/j.maturitas.2012.06.009

22 Gandhi J, Chen A, Dagur G, Suh Y, Smith N, Cali B, Khan SA. Genitourinary syndrome of menopause: an overview of clinical manifestations, pathophysiology, etiology, evaluation, and management. Am J Obstet Gynecol. 2016;215(6):704711. doi: 10.1016/j.ajog.2016.07.045

23 Espitia-De La Hoz FJ, Orozco-Gallego $\mathrm{H}$. Estriol vs estrógenos conjugados de origen equino en el tratamiento del síndrome genitourinario de la menopausia. Ginecol Obstet Mex. 2018;86(2):117-126. doi:10.24245/gom.v86i2.1881

24 Shifren JL, Gass ML; NAMS Recommendations for Clinical Care of Midlife Women Working Group. The North American Menopause Society recommendations for clinical care of midlife women. Menopause. 2014;21(10):1038-62. doi: 10.1097/GME.0000000000000319

25 Sturdee DW, Panay N; International Menopause Society Writing Group. Recommendations for the management of postmenopausal vaginal atrophy. Climacteric. 2010;13(6):509-22. doi: 10.3109/13697137.2010.522875

26 North American Menopause Society. Management of symptomatic vulvovaginal atrophy: 2013 position statement of The North American Menopause Society. Menopause. 2013;20(9):888-902; quiz 9034. doi: 10.1097/GME.0b013e3182a122c2

27 Santen RJ, Allred DC, Ardoin SP, Archer DF, Boyd N, Braunstein GD, et al. Postmenopausal hormone therapy: an Endocrine Society scientific statement. J Clin Endocrinol Metab. 2010;95(7 Suppl 1):s1-s66. doi: 10.1210/jc.2009-2509

28 De Villiers TJ, Pines A, Panay N, Gambacciani M, Archer DF, Baber RJ, et al. Updated 2013 International Menopause Society recommendations on menopausal hormone therapy and preventive strategies for midlife health. Climacteric. 2013;16(3):316-37. doi: 10.3109/13697137.2013.795683 
29 Weinberger JM, Houman J, Caron A T, Anger, J. Female Sexual Dysfunction: A Systematic Review of Outcomes Across Various Treatment Modalities. Sex Med Rev 2018;1-28. doi:10.1016/j. sxmr.2017.12.004

30 Van der Linden MC, Gerretsen G, Brandhorst MS, Ooms EC, Kremer CM, Doesburg WH. The effect of estriol on the cytology of urethra and vagina in postmenopausal women with genito-urinary symptoms. Eur J Obstet Gynecol Reprod Biol. 1993;51(1):29-33.

31 Dessole S, Rubattu G, Ambrosini G, Gallo O, Capobianco G, Cherchi PL, et al. Efficacy of low-dose intravaginal estriol on urogenital aging in postmenopausal women. Menopause. 2004;11(1) :4956. doi: 10.1097/01.GME. 0000077620. 13164.62

32 Espitia, De La Hoz, FJ, Orozco, Gallego, H, Echeverri, Ocampo, LM. Terapia hormonal y no hormonal en la vaginitis atrófica posmenopáusica: cura y satisfacción a mediano y a largo plazo de los síntomas. Rev. Col. de Menopausia. 2016;22(1): 8-17.

33 Nappi RE, Biglia N, Cagnacci A, Di Carlo C, Luisi S, Paoletti AM. Diagnosis and management of symptoms associated with vulvovaginal atrophy: expert opinion on behalf of the Italian VVA study group. Gynecol Endocrinol. 2016;32(8):602-606. doi: 10.1080/09513590.2016.1183627
34 Simon JA. Identifying and treating sexual dysfunction in postmenopausal women: the role of estrogen. J Womens Health (Larchmt). 2011;20(10):1453-65. doi: 10.1089/jwh.2010.2151

35 Lethaby A, Ayeleke RO, Roberts H. Local oestrogen for vaginal atrophy in postmenopausal women. Cochrane Database Syst Rev. 2016 Aug 31;(8):CD001500. doi: 10.1002/14651858.CD001500.pub3

36 Chism LA. Overcoming resistance and barriers to the use of local estrogen therapy for the treatment of vaginal atrophy. Int J Womens Health. 2012;4:551-7. doi: 10.2147/IJWH.S36026

37 Rahn DD, Carberry C, Sanses TV, Mamik MM, Ward RM, Meriwether KV, et al. Vaginal estrogen for genitourinary syndrome of menopause: a systematic review. Obstet Gynecol. 2014;124(6):1147-56. doi: 10.1097/AOG.0000000000000526

38 Nappi RE, Kokot-Kierepa M. Women's voices in the menopause: results from an international survey on vaginal atrophy. Maturitas. 2010;67(3):233-8. doi: 10.1016/j.maturitas.2010.08.001

39 Labrie F, Archer D, Bouchard C, Fortier M, Cusan L, Gomez JL, et al. Intravaginal dehydroepiandrosterone (Prasterone), a physiological and highly efficient treatment of vaginal atrophy. Menopause. 2009;16(5):907-22. doi: 10.1097/gme. 0b013e31819e8e2d 